\title{
Production of single top-quark final states at the LHC from supersymmetric FCNC interactions
}

\author{
David López-Val ${ }^{* a}$, Jaume Guasch ${ }^{b, c}$ and Joan Solà ${ }^{a, c}$ \\ a High Energy Physics Group, Dept. Estructura i Constituents de la Matèria, \\ Universitat de Barcelona, Av. Diagonal 647, E-08028 Barcelona, Catalonia, Spain \\ $b$ Gravitation and Cosmology Group, Dept. Física Fonamental, \\ Universitat de Barcelona, Av. Diagonal 647, E-08028 Barcelona, Catalonia, Spain \\ c Institut de Ciències del Cosmos (ICC), UB, Barcelona \\ E-mails: dlopez@ecm.ub.es, jaume.guasch@ub.edu sola@ifae.es
}

\begin{abstract}
We discuss the production of single top-quark final states by direct supersymmetric flavorchanging interactions at the LHC. The total cross section $\sigma(\mathrm{pp}(g g) \rightarrow \mathrm{t} \overline{\mathrm{c}}+\overline{\mathrm{t}} \mathrm{c})$ is computed at the 1-loop order within the unconstrained MSSM. We prove that SUSY-QCD effects may furnish sizeable production rates amounting up to $\sim 10^{5} \mathrm{t} \overline{\mathrm{c}}(\mathrm{c} \overline{\mathrm{t}})$ events per $100 \mathrm{fb}^{-1}$ of integrated luminosity, in full compliance with the stringent low-energy constraints from $b \rightarrow s \gamma$. Furthermore, we show that the cooperative SUSY-EW effects can be sizeable on their own, regardless of the SUSY-QCD contribution, with maximum production rates of the order of $10^{3}$ events per $100 \mathrm{fb}^{-1}$. Owing to the fact that FCNC production of electrically neutral heavy-quark pairs is virtually absent within the SM, we conclude that the observation of such $\mathrm{pp}(g g) \rightarrow \mathrm{t} \overline{\mathrm{c}}+\overline{\mathrm{t}} \mathrm{c}$ processes at the LHC could lead to evidence of new physics - of likely supersymmetric nature.
\end{abstract}

8th International Symposium on Radiative Corrections

October 1-5, 2007

Florence, Italy

\footnotetext{
* Speaker.
} 


\section{Introduction}

Flavor-changing neutral-current (FCNC) processes, in particular in the context of top-quark physics, constitute a fertile ground to seek for signatures of physics beyond the SM. The longknown Glashow-Iliopoulos-Maiani (GIM) mechanism is nowadays in the core of the SM, insuring the lack of tree-level FCNC interactions and also their large supression at the 1-loop order. Such supression does not fully prevent us from measuring some FCNC effects, for instance in radiative B-meson decays, $\mathrm{B} \rightarrow X_{s} \gamma$. However, the corresponding FCNC interactions involving the topquark become severly inhibited. The predicted SM branching ratios $(\mathscr{B})$ for the top-quark decays of the guise $\mathrm{t} \rightarrow \mathrm{cg}\left(\mathscr{B} \sim 10^{-11}\right)$ and $\mathrm{t} \rightarrow \mathrm{cH}\left(\mathscr{B} \sim 10^{-14}\right)$ are hopeless to be ever detected [1]. Such expected unobservability can be seen as a valuable tool for digging into traces of distinctive new physics phenomenology. For example, as soon as supersymmetric (SUSY) radiative corrections are switched on, the above picture of the FCNC top-quark dynamics may change in a dramatic way. This is the case e.g. of the top decay into MSSM neutral Higgs bosons, $h=\mathrm{h}^{0}, \mathrm{H}^{0}, \mathrm{~A}^{0}$, their branching ratios yielding $\mathscr{B}(\mathrm{t} \rightarrow \mathrm{ch}) \sim 10^{-4}$ [2], that is, 10 orders of magnitude above the SM prediction. Indeed, besides the usual SM charged-current flavor-changing interactions, the MSSM flavor dynamics is also driven by explicit intergenerational mixing terms. They stem from the fact that the squark mass matrix does not diagonalize, in general, within the same rotation matrix as for the quark mass one. In order to take such misalignment into account, a set of flavor-mixing parameters $\delta_{i j}^{(q) A B}$ is introduced, being defined in such a way that the soft-SUSY breaking squark mass matrix elements of type $q=u(d), i(j)$-th flavor $(i \neq j)$ and $A(B)$-chirality read $\left(M_{i j}^{(q) A B}\right)^{2}=$ $m_{i} m_{j} \delta_{i j}^{(q) A B}{ }_{1}$. Henceforth we will only allow non-zero mixing terms of LL type as this possibility is the simplest one and is well motivated by renormalization group arguments [3]. The presence of such explicit flavor-mixing parameters may provide non-supressed sources of non-standard FCNC phenomena.

\section{Numerical results}

In what follows we shall discuss the full MSSM contribution (including the SUSY-QCD and the SUSY-EW effects) to $\sigma(\mathrm{pp}(g g) \rightarrow \mathrm{t} \overline{\mathrm{c}}+\overline{\mathrm{t}} \mathrm{c})$, where $(\mathrm{gg})$ denote gluon-fusion. The latter is (by far) the leading production mechanism at the partonic level. In an obvious notation, we have $\sigma=2 \sigma_{\mathrm{tc}}$. Throughout the present work we have made use of the standard algebraic and numerical packages FeynArts, FormCalc and LoopTools [4], together with the program HadCalc [5], for the computation of the hadronic cross sections.

Let us begin by adressing the SUSY-QCD contribution to $\sigma(\mathrm{pp}(g g) \rightarrow \mathrm{t} \overline{\mathrm{c}}+\overline{\mathrm{t}} \mathrm{c})$. In a general scenario within non-vanishing flavor-mixing, the FCNC interactions are induced by gluino-quarksquark vertices and lead to partonic cross sections of the following (approximate) form ${ }^{2}$ :

$$
\sigma(\mathrm{gg} \rightarrow \mathrm{t} \overline{\mathrm{c}}) \sim \frac{\left|\delta_{23}^{(u) L L}\right|^{2}}{s}\left(\frac{\alpha_{s}^{2}}{16 \pi^{2}}\right)^{2} \frac{m_{t}^{2}\left(A_{t}-\mu / \tan \beta\right)^{2}}{M_{\mathrm{SUSY}}^{4}} .
$$

\footnotetext{
${ }^{1}$ We shall settle the soft-SUSY breaking terms to a common mass scale, $m_{i}=M_{S U S Y}, \forall i$.

${ }^{2}$ We use a standard notation for the SUSY parameters, see e.g. [9] and references therein for the concrete definitions.
} 
Let us observe that the same sort of gluino-mediated FCNC interactions will also induce SUSYQCD corrections to the low-energy observable $\mathscr{B}_{\text {exp }}(\mathrm{b} \rightarrow \mathrm{s} \gamma)$ [6]. Enforcing the predicted $\mathscr{B}_{M S S M}(\mathrm{~b} \rightarrow$ $\mathrm{s} \gamma)$ to stay within the experimental bounds $\left([2-4.5] \times 10^{-4}\right.$ at the $3 \sigma$ level [7]), we will avoid artificially enhanced flavor-mixing sources. Moreover, due to the $S U(2)_{L}$ gauge invariance, the LLblocks of the soft-SUSY breaking squark masses get tied through $\left(M^{(u) L L}\right)^{2}=K\left(M^{(d) L L}\right)^{2} K^{\dagger}$, in such a way that the $\mathrm{b} \rightarrow \mathrm{s} \gamma$ bound on $\delta_{23}^{(d) L L}$ translates into a bound on $\delta_{23}^{(u) L L}$. Our endeavor is to systematically seek for those (experimentally allowed) regions in the MSSM parameter space where the SUSY-QCD effects are maximum. We can manage to project this optimal regime by means of an approximate analytical procedure. To begin with, let us define $\delta_{33}^{(u) L R}=m_{t}\left(A_{t}-\right.$ $\mu / \tan \beta) / M_{S U S Y}^{2}$, from which we can rewrite Eq. (2.1) as $\sigma(\mathrm{gg} \rightarrow \mathrm{t} \overline{\mathrm{c}})=\left(\delta_{23}^{(u) L L}\right)^{2}\left(\delta_{33}^{(u) L R}\right)^{2} / s$. Notice that isolines of constant $\sigma=\sigma_{0}$ are hyperbolae in the $\delta_{23}^{(u) L L} \times \delta_{33}^{(u) L R}$ plane. On the other hand, upon diagonalization of the squark mass matrix, the (approximate) value of the lightest squark mass reads $m_{\tilde{q}_{1}}=M_{S U S Y}^{2}\left(1-\sqrt{\left(\delta_{23}^{(u) L L}\right)^{2}+\left(\delta_{33}^{(u) L R}\right)^{2}}\right)$. The LEP mass bounds $\left(m_{\tilde{q}_{1}} \geq 90 \mathrm{GeV}\right)$ yield the inequality $\left(\delta_{23}^{(u) L L}\right)^{2}+\left(\delta_{33}^{(u) L R}\right)^{2}<1-m_{\tilde{q}_{1}}^{2} / M_{S U S Y}^{2} \equiv R^{2}$. We further impose that $\left|A_{t}\right|<3 M_{S U S Y}$ in order to avoid color-breaking minima, thus supplying a new restriction on $\delta_{33}^{(u) L R}$. Now it is simply a matter of picking out the point in the bisector line $\delta_{33}^{(u) L R}=\delta_{23}^{(u) L L}$ where the outermost hyperbola is tangent to the circle of radius $R$ (see right-panel of Figure 1). The desired maximum, then, is:

$$
\delta_{23}^{(u) L L}=\frac{\sqrt{2}}{1+\left[1+\frac{2}{9} m_{\tilde{q}_{1}}^{2} / m_{t}^{2}\right]^{1 / 2}} \simeq 0.7,
$$

from which we also find $A_{t} \simeq 2238 \mathrm{GeV}$ and $M_{S U S Y} \simeq 746 \mathrm{GeV}$. A complete analysis of the behavior of the cross section in the SUSY-QCD favored regime can be found in $[8,9]$. Therein it is shown that, for relatively light gluino masses (of, say, $m_{\tilde{g}} \sim 200 \mathrm{GeV}$ ) the total cross section can reach $\sigma \sim 1 \mathrm{pb}$, that is, around $10^{5} \mathrm{t} \overline{\mathrm{c}}(\mathrm{c} \overline{\mathrm{t}})$ events per $100 \mathrm{fb}^{-1}$ of integrated luminosity. In view of the fact that the SM contribution to this process reads $\sigma(\mathrm{pp}(g g) \rightarrow \mathrm{t} \overline{\mathrm{c}}+\overline{\mathrm{t}} \mathrm{c})=8.46 \times 10^{-8} \mathrm{pb}$, the maximum SUSY enhancement is some 7 orders of magnitude larger and could thus provide a measurable signature at the $\mathrm{pb}$ level.

The SUSY-EW contributions to $\mathrm{pp}(g g) \rightarrow \mathrm{t} \overline{\mathrm{c}}+\overline{\mathrm{t}} \mathrm{c}$ are triggered by a large set of loop diagrams involving charginos, neutralinos and charged Higgs bosons (see Ref. [9] for details). If gluinos are very heavy $\left(m_{\tilde{g}} \sim 2 \mathrm{TeV}\right)$, or the FCNC parameter $\delta_{23}^{(u) L L}$ very small, it may be interesting to explore the behavior of $\sigma_{\mathrm{t} \overline{\mathrm{c}}}$ in a SUSY-EW favored scenario. Such a regime can be achieved essentially by arranging a choice of MSSM parameters that insures relatively light neutralinos, charginos (within their respective LEP mass bounds, $m_{\tilde{\chi}_{1}^{0}} \geq 46 \mathrm{GeV}, m_{\tilde{\chi}_{1}^{ \pm}} \geq 96 \mathrm{GeV}$ ), together with light charged Higgs bosons and squarks (mainly stops), and of course in compliance with the $\mathrm{b} \rightarrow \mathrm{s} \gamma$ restrictions. The resulting behavior of $\sigma_{\mathrm{t} \overline{\mathrm{c}}}$ as a function of $\left.\mathbf{a}\right) \tan \beta$ and $\left.\mathbf{b}\right) A_{t}$ is presented in the upper-left panels of Figure 1.

In the lower-left panels of Figure 11 we also quote the $\sigma_{\mathrm{tc}}$ curves as a function of $\delta_{23}^{(u) L L}$ for both c) SUSY-QCD favored and d) SUSY-EW favored regimes. While in the former the gluinomediated contribution (with $m_{\tilde{g}}=200 \mathrm{GeV}$ ) is largely dominant (namely, it lies at least 4 orders of magnitude over the chargino curve), with the highest production rates reaching the level of $\sim 10^{5}$ events per $100 \mathrm{fb}^{-1}$, the situation is substantially different in the latter scenario. Therein we find 
a)

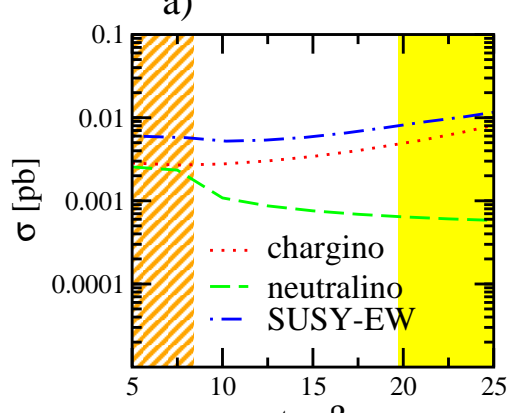

c)

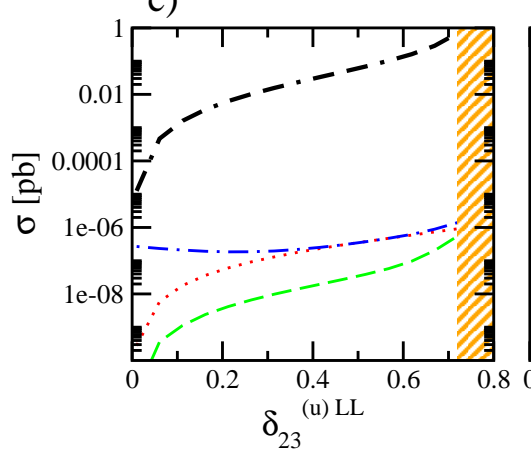

b)

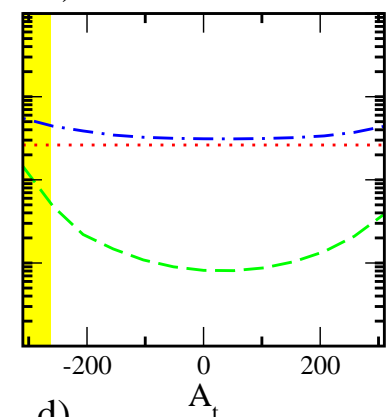

d)

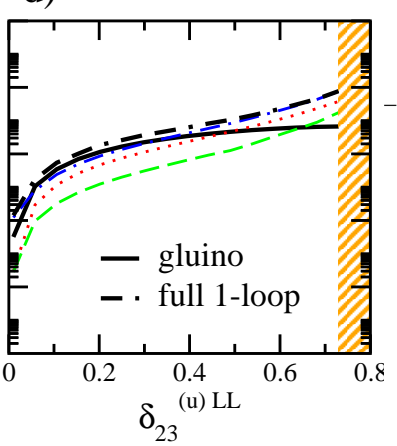

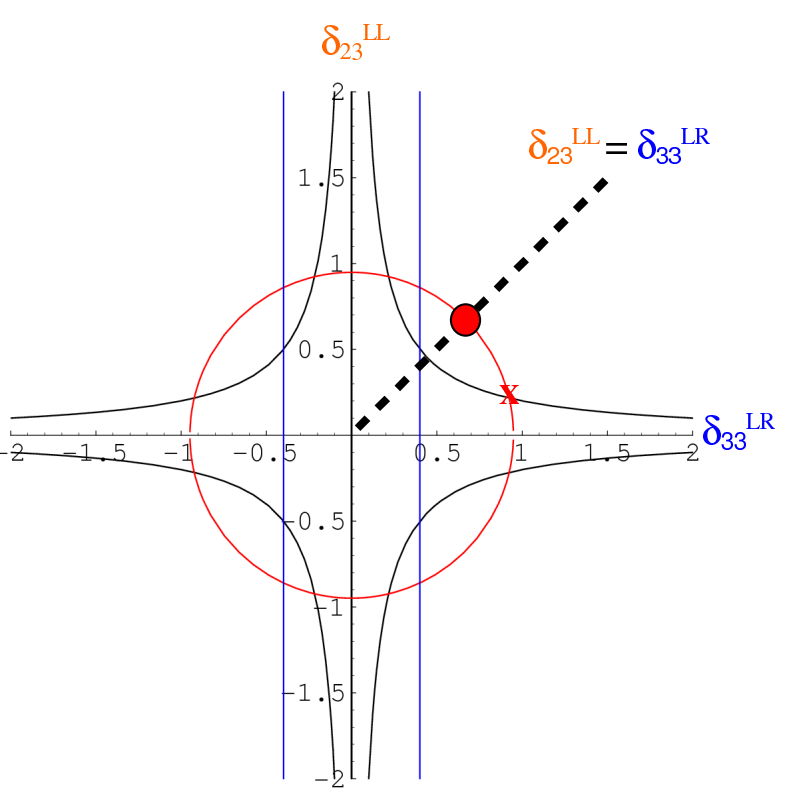

Figure 1: (Upper-left panels) SUSY-EW contribution to $\sigma_{\mathrm{t} \overline{\mathrm{c}}}$ (in pb) as a function of a) $\tan \beta$ and $\left.\mathbf{b}\right) A_{t}$. (Lower-left panels) Combined SUSY-QCD and SUSY-EW contributions to $\sigma_{\mathrm{tc}}(\mathrm{in} \mathrm{pb})$ as a function of $\delta_{23}^{(u) L L}$ within c) a SUSYQCD favored and d) a SUSY-EW favored scenario. The shaded regions are excluded by $\mathrm{b} \rightarrow \mathrm{s} \gamma$ restrictions, while the dashed ones are ruled out by the mass bounds on the lightest supersymmetric particles. (Right panel) Sketch of the geometrical configuration leading to the optimal SUSY-QCD contribution (see the text for details).

light neutralinos and charginos (close to their mass bounds) meeting together with rather heavy $\left(m_{\tilde{g}}=2 \mathrm{TeV}\right)$ gluinos. It is remarkable that even for moderate values of $\delta_{23}^{(u) L L}$, the SUSY-EW effects turn out to be significant, e.g. for $\delta_{23}^{(u) L L} \sim 0.4$ the full one-loop curve is enhanced by a factor 2 with respect to the SUSY-QCD contribution. The larger the flavor-mixing is, the larger the SUSY-EW effects become, achieving maximal values that amount up to roughly $\sigma=2 \sigma_{\mathrm{t} \overline{\mathrm{c}}} \sim 0.01 \mathrm{pb}$ (1 order of magnitude above the SUSY-QCD contribution in this alternate region of the parameter space).

\section{Discussion and conclusions}

We have discussed the supersymmetric production of single top-quark final states at the LHC. Besides the fact that we only allow explicit sources of flavor-mixing in the LL-block of the squark mass matrix, the inclusion of the interplay between the top-quark physics and the stringent lowenergy $\mathrm{b} \rightarrow \mathrm{s} \gamma$ constraints, provides a self-consistent framework to carry out the computation of $\sigma(\mathrm{pp}(g g) \rightarrow \mathrm{t} \overline{\mathrm{c}}+\overline{\mathrm{tc}})=2 \sigma_{\mathrm{t} \overline{\mathrm{c}}}$. Related supersymmetric studies of SUSY-FCNC processes in the literature can be found e.g. in [2,8-11] and references therein. In this note we have revisited the SUSY-QCD analysis of $\sigma_{\mathrm{t} \overline{\mathrm{c}}}$ presented in [8], which was extended by the inclusion of the SUSY$\mathrm{EW}$ effects in [9]. See also the latter reference for the potential impact of the $\mathrm{B}_{s}^{0}-\overline{\mathrm{B}}_{s}^{0}$ mixing restrictions. Here we have singled out only the regions of the MSSM parameter space where the SUSY-QCD contribution becomes optimal, in full agreement with the stringent and robust 
experimental restrictions imposed by $\mathrm{b} \rightarrow \mathrm{s} \gamma$. The most favorable regimes are attained in the case of large intergenerational mixing and relatively light gluinos, in which we get cross sections up to $\sim 1 \mathrm{pb}$ - barely $10^{5}$ events per $100 \mathrm{fb}^{-1}$ of integrated luminosity. Furthermore, we have also proved the existence of configurations in the MSSM parameter space for which the SUSY-EW effects become fully competitive with respect to the SUSY-QCD ones. It means that for virtually decoupled gluinos (and so a largely supressed SUSY-QCD contribution) the overall cross section may still be sizeable thanks to the cooperative SUSY-EW flavor-changing interactions mediated by charginos and neutralinos. Such regimes entail maximum cross sections of the order of $\sim 0.01 \mathrm{pb}$ - 1000 events per $100 \mathrm{fb}^{-1}$. Worth noticing is that other alternative sources of $t \bar{c}(c \bar{t})$ final states in either the MSSM [11] or the Two-Higgs-doublet model [12] are not competitive with the direct SUSY mechanism $\mathrm{pp}(g g) \rightarrow \mathrm{t} \overline{\mathrm{c}}+\overline{\mathrm{t}} \mathrm{c}$. The latter might thus be, by far, the most efficient mechanism of $t \bar{c}(\bar{c} \bar{t})$ FCNC production within renormalizable extensions of the SM. Being the corresponding SM contribution virtually absent, the presence of such $t \bar{c}(c \bar{t})$ final states, if effectively tagged and confidently isolated from the background, could lead to evidence of supersymmetric physics.

Acknowledgments DLV has been supported by the MEC FPU grant Ref. AP2006-00357; JG and JS in part by MEC and FEDER under project 2004-04582-C02-01; JS also by DURSI Generalitat de Catalunya under project 2005SGR00564. JG is thankful to the Universidad de Zaragoza, for their kind hospitality.

\section{References}

[1] J.L. Díaz-Cruz, R. Martínez, M.A. Pérez and A. Rosado, Phys. Rev. D 41 ( 891) 1990; G. Eilam, J.L. Hewett and A. Soni, Phys. Rev. D 44 ( 1991) 1473, Erratum-ibid D59(1999) 039901; B. Mele, S. Petrarca and A. Soddu, Phys. Lett. B 435 (1998) 401.

[2] J. Guasch and J. Solà, Nucl. Phys. B 562 ( 1999) 3.

[3] M.J. Duncan, Nucl. Phys. B 221 ( 1983) 285; Phys. Rev. D 301 ( 1985) 1139.

[4] T. Hahn, FeynArts 3.2, FormCalc and LoopTools user's guides, available from http: / / www. feynarts. de; T. Hahn, Comput. Phys. Commun. 168 (2005) 78.

[5] M. Rauch, PhD thesis, Technische Universitat Muenchen (2006).

[6] C. Bobeth, M. Misiak, J. Urban, Nucl. Phys. B 567 ( 2000) 153.

[7] B. Aubert et al., BaBar Collaboration [hep-ex/0207076].

[8] J. Guasch, W. Hollik, S. Peñaranda, J. Solà, Nucl. Phys. Proc. Supp. 157 (2006) 152.

[9] D. López-Val, J. Guasch and J. Solà, JHEP 712 (2007) 54.

[10] J.J. Liu, C.S Li, L.L. Yang and L.G. Jin, Nucl. Phys. B 705 ( 2005) 3; G. Eilam, M. Frank and I. Turan, Phys. Rev. 774 ( 2006) 0305012; J. J. Cao, G. Eilam, M. Frank, K. Hikasa, G. L. Liu, I. Turan and J.M. Yang, Phys. Rev. $\mathrm{D} 75$ ( 2007) 075021; J. L. Díaz-Cruz, H.-J. He and C. P. Yuan, Phys. Lett. B 530 (2002) 179.

[11] S. Béjar, J. Guasch and J. Solà, JHEP 510 (2005) 113; Nucl. Phys. Proc. Supp. 157 (2006) 147; S. Béjar, F. Dilmé, J. Guasch and J. Solà, JHEP 408 (2004) 18.

[12] S. Béjar, J. Guasch and J. Solà, Nucl. Phys. B 600 ( 2001) 21; [hep-ph/0101294]; Nucl. Phys. B 675 ( 2003 ) 270; A. Arhrib, Phys. Lett. B 612 (2005) 263; Phys. Rev. D 72 ( 2005) 075016. 